\title{
Constellation Shaping for Bit-Interleaved Coded APSK
}

\author{
Matthew C. Valenti and Xingyu Xiang \\ Lane Department of Computer Science and Electrical Engineering \\ West Virginia University, Morgantown, WV, U.S.A. \\ Email: valenti@ieee.org, xiang.xxy2008@gmail.com
}

\begin{abstract}
This paper considers a technique for shaping a turbo-coded amplitude-phase shift keying (APSK) constellation. After bit-interleaving, a subset of the bits output by a binary turbo encoder are passed through a nonlinear shaping encoder. The bits at the output of the shaping encoder are more likely to be a zero than a one. These "shaping" bits are interleaved and used to select from among a plurality of subconstellations, while the unshaped bits are used to select the symbol within the subconstellation. Symbols from lower-energy subconstellations are selected more frequently than those from higher-energy subconstellations. Information rates are computed for a variety of APSK and shaping code parameters, in an effort to optimize these parameters. It is found that, in theory, shaping gains of slightly over $0.3 \mathrm{~dB}$ may be achieved with 16-APSK and 32-APSK in AWGN. Simulation results show the bit error performance of the constellation-shaping strategy with an actual turbo code. The BER results suggest that gains beyond $0.3 \mathrm{~dB}$ may be possible in AWGN when an iterative decoder is used, presumably due to the additional coding gain of the shaping code.
\end{abstract}

\section{INTRODUCTION}

Amplitude phase-shift keying (APSK) is a modulation consisting of several concentric rings of signals, with each ring containing signals that are separated by a constant phase offset. APSK has recently become widely adopted, due primarily to its inclusion in the second generation of the Digital Video Broadcasting Satellite standard, DVB-S2 [1], as well as some other standards such as DVB-SH, IPoS, GMR-2 3G, and ABS$\mathrm{S}$. APSK is known to be both spectral and energy efficient, and is especially well suited for nonlinear channels. For a given modulation order $M$, an APSK constellation is characterized by the number of rings, the number of signals in each ring, the relative radii of the rings, and the phase offset of the rings relative to each other. In [2] and [3] these parameters were optimized using a technique based on information theory. In particular, the achievable information rate was computed for each choice of APSK parameters, and from these information rates, the optimal parameters were identified. The information rate can then be used to accurately predict the minimum received signal-to-noise ratio (SNR) required for a capacityapproaching code, such as a turbo or LDPC code, to achieve an arbitrarily low bit error rate.

The information rates used in [2] to optimize the modulation parameters were computed by assuming that the $M$ symbols are selected with uniform probability. This is indeed true for

This work was supported in part by the National Science Foundation under Award No. CNS-0750821. many operational systems, such as DVB-S2. However, these achievable information rates are not equal to the capacity of the channel, because achieving capacity requires that the optimization permit a nonuniform distribution of symbols. In [3], a nonuniform symbol distribution was considered and optimal symbol probabilities were determined by maximizing the mutual information. However, [3] does not specify how these distributions can be achieved in a practical system.

While it is difficult to induce an arbitrary symbol distribution, constellation shaping techniques, such as the ones proposed in [4], have been devised that bias the symbols in an effort to increase the information rate. When constellationshaping is combined with powerful, capacity-approaching codes, the method of combination is a challenging problem. In [5] and [6], Le Goff et al. propose a shaping technique suitable for bit-interleaved systems typical of modern communication standards. The technique involves the use of the short nonlinear shaping codes described in [4] to select from among a plurality of subconstellations. The constellation is partitioned into subconstellations such that subconstellations with lower average energy will be selected more frequently than constellations with higher energy. However, the results in [5] are limited to convolutionally-coded 16-QAM, while the results in [6] are limited to turbo-coded PAM.

In this paper, we draw inspiration from the informationtheoretic optimization of APSK with given in [2], [3] and the practical shaping technique proposed in [5], [6]. We use information-theory to jointly optimize parameters used by both the APSK constellation and the shaping code. Once the parameters are identified, we implement the system with actual turbo and shaping codes, and can confirm through bit-error rate curves that shaping does indeed improve performance. From one perspective, our work can be seen as an extension of [2] to the case of a nonuniform input distribution. From another perspective, it can be seen as an application of [5] and [6] to APSK modulation. Alternatively, it can be considered as an adaption of the optimization approach of [3] to the shaping strategy of [5] and [6].

We optimize our system for the AWGN channel over a discrete set of possible signal constellation designs and shaping codes. Because the optimization was performed over a set of 11 constellations ( 32-APSK constellations) using 121 distinct shaping codes, a total of 1,331 sets of parameters was considered. This was 


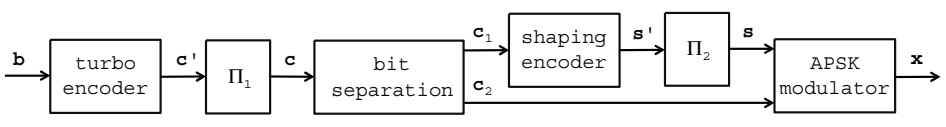

Fig. 1. Transmitter structure.

a computationally challenging task that required the use of a 64-core cluster computer, and it is our hope that the results we found will prove useful to engineers who seek to enhance the performance of APSK-based systems.

The remainder of this paper is organized as follows. A model for bit-interleaved coded APSK with constellation shaping is given in Section II. The section describes the operation of the nonlinear shaping encoder. In Section III, constellation shaping strategies are proposed for constellations with mappings that are equivalent (up to a bitwise complement) to those in the DVB-S2 standard. The shaping and modulation parameters are optimized using an information-theoretic approach in Section IV. The implementation of an actual coded system is discussed in Section V. Finally, conclusions are given in VI.

\section{SYSTEM MODEL}

A block diagram of the transmitter is shown in Fig. 1. The input to the system is a length- $K$ vector $\mathbf{b}$ of information bits. The information bits are encoded by a rate $R_{c}=K / N_{c}$ binary turbo encoder to produce the length- $N_{c}$ codeword $\mathbf{c}^{\prime}$. The codeword is permuted by a first interleaver $\Pi_{1}$ to produce the vector $\mathbf{c}$. The bits in $\mathbf{c}$ are separated into two disjoint groups, $\mathbf{c}_{1}$ of length $N_{1}=K_{s}$ and $\mathbf{c}_{2}$ of length $N_{2}=N_{c}-K_{s}$, where $K_{s}$ is the number of bits at the input to the shaping encoder. Because of interleaver $\Pi_{1}$, the way that the bits are separated is arbitrary. The vector $\mathbf{c}_{1}$ is passed through a rate $R_{s}=K_{s} / N_{s}$ shaping encoder, whose implementation is discussed starting in the next paragraph, to produce the length- $N_{s}$ vector of shaping bits $\mathbf{s}^{\prime}$. The shaping bits $\mathbf{s}^{\prime}$ are permuted by a second interleaver $\Pi_{2}$ to produce the vector $\mathbf{s}$. The $N_{s}$ bits in $\mathbf{s}$ and $N_{2}$ bits in $\mathbf{c}_{2}$ are input to the APSK modulator. The modulator produces a length- $N$ vector $\mathbf{x}$ of modulated symbols drawn from the constellation $\mathcal{X}$ with $|\mathcal{X}|=M=2^{m}$. Let $g<m$ be the number of shaping bits per symbol. Each symbol in $\mathrm{x}$ is selected from $\mathcal{X}$ according to the prescribed symbol mapping by using $g$ bits from $\mathbf{s}$ and $m-g$ bits from $\mathbf{c}_{2}$. Strategies for mapping bits to symbols to achieve the desired shaping are discussed in Section III.

Let $p_{0}$ denote the probability that a particular bit in $\mathbf{s}$ (or, equivalently, $\mathbf{s}^{\prime}$ ) is equal to zero, and $p_{1}$ be the probability that it is equal to one. The purpose of the shaping code is to produce an output with a particular $p_{0}>1 / 2$. The shaping code must necessarily be nonlinear, and can be decoded by exhaustively comparing the received codeword against all the codewords according to the MAP algorithm [6]. Because of the "brute-force" decoder implementation, it is advantageous to break the shaping encoder input $\mathbf{c}_{1}$ into $L$ short blocks, and encode each block with a small $\left(n_{s}, k_{s}\right)$ nonlinear block code, where $K_{s}=L k_{s}$, and then reassemble the $L$ output blocks into the length $N_{s}=L n_{s}$ shaping codeword s'. We assume that $L$ is an integer multiple of $g$.

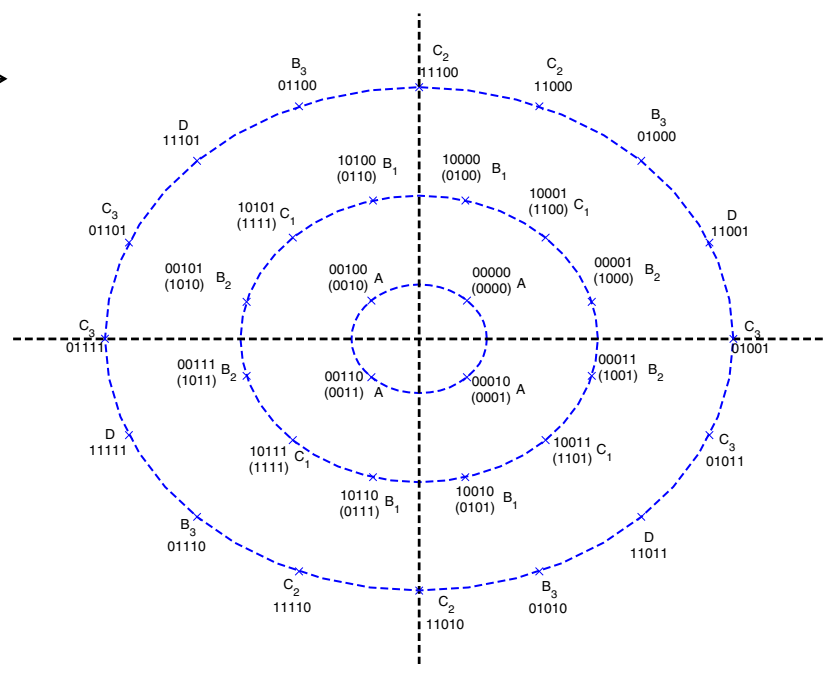

Fig. 2. APSK constellations. 16-APSK uses the signals shown in the two innermost rings with symbol mappings indicated in parenthesis. 32-APSK uses all of the shown signals.

The short $\left(n_{s}, k_{s}\right)$ shaping code $\mathcal{S}$ is constructed according to the methodology of [4], which we summarize as follows. The goal of the construction process is for $\mathcal{S}$ to contain $2^{k_{s}}$ distinct codewords of lowest possible Hamming weight. Construction is a recursive process, with $\mathcal{S}$ initialized to contain the all-zeros codeword of length $n_{s}$. Codewords of higher and higher weight are recursively added to $\mathcal{S}$ until $|\mathcal{S}|=2^{k_{s}}$. Suppose that $\mathcal{S}$ contains all codewords of weight $w-1$ or lower. During the next recursion, a codeword of weight $w$ is drawn at random, and if it has not already been drawn, it is added to $\mathcal{S}$. Weight $w$ codewords are repetitively drawn and added to $\mathcal{S}$ until either the number of distinct codeword in $\mathcal{S}$ is $2^{k_{s}}$ or all weight $w$ codewords have been used. In the former case, the code construction is complete, while in the latter case it moves on to begin adding codewords of weight $w+1$.

As an example, consider the $\left(n_{s}, k_{s}\right)=(9,7)$ code. The number of codewords in $\mathcal{S}$ is $2^{7}=128$. There are

$$
\left(\begin{array}{l}
9 \\
0
\end{array}\right)+\left(\begin{array}{l}
9 \\
1
\end{array}\right)+\left(\begin{array}{l}
9 \\
2
\end{array}\right)+\left(\begin{array}{l}
9 \\
3
\end{array}\right)=174
$$

binary 9-tuples of weight three or less. It follows that $\mathcal{S}$ is a subset of these 9-tuples. In particular, $\mathcal{S}$ will contain all 9tuples of weight- 2 or less, and will contain 38 of the weight-3 9-tuples. Due to the presence of the second interleaver $\Pi_{2}$, the choice of which weight-3 9-tuples to use is not important.

The overall rate of the system $R=K / N$ is the number of information bits per modulated symbol, and is related to the rates of the turbo and shaping codes by:

$$
R=R_{c}\left[m+g\left(R_{s}+1\right)\right] .
$$

When shaping is used, $g>0$ and $R_{s}<1$, which implies that for a fixed $R$, the rate of the turbo code $R_{c}$ with shaping must be higher than the rate of the turbo code when shaping is not used. In Section IV we investigate the tradeoff between $R_{c}$ and $R_{s}$ by computing information rates of the shaped constellations. 


\section{Shaping STRATEgIES}

\section{A. Shaping for 16-APSK}

Consider the 16-APSK constellation, which uses the two innermost rings shown in Fig. 2. The inner ring contains 4 points, while the outer ring contains 12 points. The bit mapping is as indicated on the figure. Note that this constellation is identical to the 16-APSK constellation in the DVB-S2 standard [1] and the mapping is identical except that the first two bits are complemented. The ratio of the radius of the outer ring to the radius of the inner ring is denoted $\gamma$, which according to the DVB-S2 standard must assume a value of $2.57,2.60$, $2.70,2.75,2.85$, or 3.15 .

The minimum size partition in the shaping scheme should be equal to the number of minimum-energy signals, which in this case is four. It follows that the number of shaping bits may be either $g=1$ or $g=2$. In the case of $g=1$ shaping bit, the shaping bit is the first bit of the four-bit word labeling the constellation points. The signal set is partitioned into two sets. The first set contains points labeled $A$ and $B_{1}$ in Fig. 2 , while the second set contains points labeled $B_{2}$ and $C_{1}$ in Fig. 2. The first set is selected with probability $p_{0}$, while the second set is selected with probability $p_{1}$.

In the case that $g=2$, the shaping bits are the first two bits of the word. The signal set is partitioned into the four sets indicated in Fig. 2, i.e. $A, B_{1}, B_{2}$, and $C_{1}$. Set $A$ is selected with probability $p_{0}^{2}$, set $B_{1}$ and $B_{2}$ are each selected with probability $p_{0} p_{1}$, and set $C_{1}$ is selected with probability $p_{1}^{2}$. As $p_{0}>p_{1}$, it follows that signals in set $A$ are selected most often, and signals in set $C_{1}$ are selected least often.

\section{B. Shaping for 32-APSK}

If all the signal points shown in Fig. 2 are used, then the modulation is 32-APSK. The constellation consists of three concentric rings, with 4 points in the inner ring, 12 points in the middle ring, and 16 points in the outer ring. This constellation is identical to the 32-APSK constellation in the DVB-S2 standard [1] and the mapping is identical except that the first and last bits are complemented. The ratio of the radius of the middle ring to the radius of the inner ring is denoted $\gamma_{1}$, while the ratio of the radius of the outer ring to the radius of the inner ring is denoted $\gamma_{2}$. According to the standard, the pair $\left\{\gamma_{1}, \gamma_{2}\right\}$ must be one of the following: $\{2.53,4.30\}$, $\{2.54,4.33\},\{2.64,4.64\},\{2.72,4.87\}$, or $\{2.84,5.27\}$.

As with 16-APSK, the number of minimum energy signals is four, which is the size of the smallest partition. The number of shaping bits should be no more than three. In the case of $g=1$ shaping bit, the shaping bit is the second bit of the word. This divides the constellation into two partitions. The first partition, which is selected with probability $p_{0}$, contains the signals in the first two rings (i.e. sets $A, B_{1}, B_{2}$, and $C_{1}$ ). The second partition, which is selected with probability $p_{1}$, contains the signals in the outer ring (i.e. sets $B_{3}, C_{2}, C_{3}$, and $D$ ). With this scheme, signals in the first two rings are more likely to be selected than signals in the outer ring.

When $g=2$, the shaping bits are the second and last bits of the word. The signal set is partitioned into four sets: $\left\{A, B_{1}\right\}$,
$\left\{B_{2}, C_{1}\right\},\left\{B_{3}, C_{2}\right\}$, and $\left\{C_{3}, D\right\}$. The partition $\left\{A, B_{1}\right\}$ is selected with probability $p_{0}^{2}$, making it most likely to be selected. The partition $\left\{C_{3}, D\right\}$ is selected with probability $p_{1}^{2}$, making it least likely to be selected. The other two partitions are each selected with probability $p_{0} p_{1}$.

When $g=3$, the shaping bits are the first, second, and last bits of the word. The signal set is partitioned into the eight sets indicated in Fig. 2, i.e. $A, B_{1}, B_{2}, B_{3}, C_{1}, C_{2}, C_{3}$, and $D$. Partition $A$ is most likely and is selected with probability $p_{0}^{3}$. The $B_{k}$ partitions are selected with probability $p_{0}^{2} p_{1}$, while the $C_{k}$ partitions are selected with probability $p_{0} p_{1}^{2}$. Finally, the $D$ partition is selected with probability $p_{1}^{3}$, which makes it least likely to be selected.

\section{PARAMETER Optimization}

With the shaping techniques described in the previous section, energy may be conserved by using larger values of $p_{0}$. However, larger values of $p_{0}$ generally require lower $R_{s}$, the rate of the shaping code. To maintain a fixed overall rate $R$, using a lower $R_{s}$ requires a larger value of $R_{c}$, which weakens the effectiveness of the turbo code. Clearly there is a tradeoff between $R_{s}$ and $R_{c}$. To determine the optimum tradeoff, we turn to information theory.

Let $Y$ be the output of an additive white Gaussian noise (AWGN) channel with complex scalar input $X \in \mathcal{X}$. The capacity of the channel is [7]

$$
C=\max _{p(x)} I(X ; Y),
$$

where $p(x)$ is the probability mass function of $X$, the information rate (also called average mutual information) is

$$
I(X ; Y)=E[i(X ; Y)],
$$

and

$$
i(x ; y)=\log \frac{p(x, y)}{p(x) p(y)}=\log \frac{p(y \mid x)}{p(y)} .
$$

Note that the expectation in (3) is with respect to the joint pdf $p(x, y)$. When a base-2 logarithm is used, then (2) has units of bits per symbol. From the theorem on total probability,

$$
p(y)=\sum_{x^{\prime} \in \mathcal{X}} p\left(y \mid x^{\prime}\right) p\left(x^{\prime}\right) .
$$

Substituting (5) into (4) gives

$$
i(x ; y)=\log p(y \mid x)-\log \left(\sum_{x^{\prime} \in \mathcal{X}} p\left(y \mid x^{\prime}\right) p\left(x^{\prime}\right)\right) .
$$

For the complex Gaussian noise channel,

$$
p(y \mid x) \propto \exp \left(-\frac{\mathcal{E}_{s}}{N_{0}}|y-x|^{2}\right)
$$

where $\mathcal{E}_{s}$ is the energy per modulated symbol and $N_{0}$ is the one-sided noise-spectral density. It follows that

$$
\log p(y \mid x)=-\frac{\mathcal{E}_{s}}{N_{0}}|y-x|^{2}+\kappa
$$




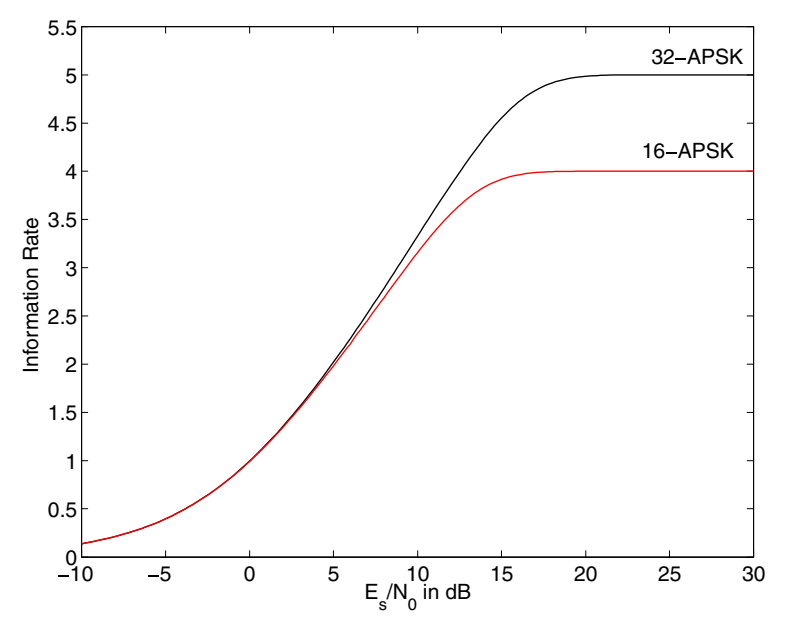

Fig. 3. Information rate (in bits per channel use) of 16-APSK and 32APSK with uniform input distributions over an AWGN channel. The rates are maximized over the permissible ring radius ratios.

Where the constant $\kappa$ will cancel in the log-likelihood ratio of (6).

The capacity given in (2) is found by maximizing the information rate with respect to the distribution of the input $X$. For a general $M$-ary modulation scheme, the optimization requires that the probability of occurrence of each of the symbols be independently varied. Techniques for finding the optimizing distributions may be readily found in the literature, such as the Blahut-Arimoto algorithm [8]. However, the shaping scheme proposed in Section III does not allow for independent symbol probabilities. Rather, the symbol probabilities are correlated by the value of $p_{0}$, the number of shaping bits, and the symbol mapping pattern.

Rather than allowing arbitrary $p(x)$, we optimize our system under the constraint of the proposed shaping techniques. For a particular constellation $\mathcal{X}$, number of shaping bits $g$, and $\left(n_{s}, k_{s}\right)$ shaping code, we numerically evaluate the information rate $I(X ; Y)$. While the evaluation could be done using a Monte Carlo integration [9], we use the Gauss-Hermite quadratures method of evaluation described in [10].

We begin by evaluating the information rates under the assumption that the input symbols have a uniform distribution (also called the symmetric information rate). For $M=16$, we evaluated the information rate as a function of $\mathcal{E}_{s} / N_{0}$ for each of the six values of $\gamma$ specified in the DVB-S2 standard. For each $M$ and each value of $\mathcal{E}_{s} / N_{0}$, we note the maximum value of the information rate over all choices of $\gamma$. Similarly, for $M=32$, we evaluated the information rate for each of the five values of $\left\{\gamma_{1}, \gamma_{2}\right\}$ specified in the DVB-S2 standard, and we noted the maximum information rate at each $\mathcal{E}_{s} / N_{0}$. The information rate, maximized over the permissible ring radius ratios, is shown for the uniform-input case in Fig. 3.

Next, we compute information rates when shaping is used. For each constellation $\mathcal{X}$, we consider all shaping codes with $n_{s} \leq 20$ and $k_{s} \leq 10$. Larger values are not considered due to their high decoding complexity (which is exponential in $k_{c}$ ). For each shaping code, we determine the corresponding value
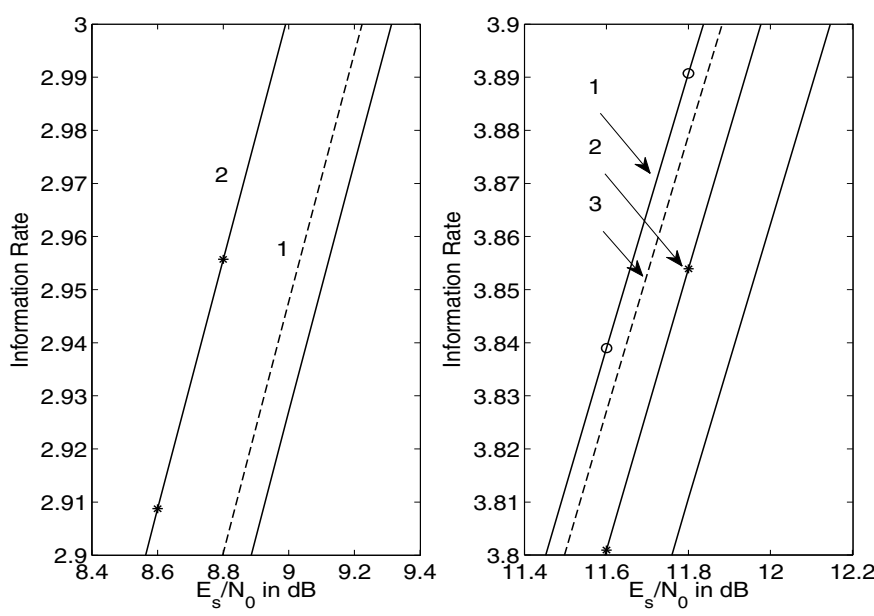

Fig. 4. Information rate of nonuniform 16-APSK (subfigure on the left) and 32-APSK (subfigure on the right) over an AWGN channel. The number of shaping bits used is indicated. The solid black line on the right of each subfigure corresponds to a uniform input distribution.

of $p_{0}$. For a given number of shaping bits $g$ and the shaping strategy given by Section III, we determine the pmf $p(x)$ and compute the corresponding information rate through numerical integration. The total number of distinct $p_{0}$ that we consider is 121 , and we repeat this exercise for each permissible value of $\gamma$ (if $M=16$ ) or $\left\{\gamma_{1}, \gamma_{2}\right\}$ (if $M=32$ ). For each $M$ and $g$, we determine the maximum information rate at each value of $\mathcal{E}_{s} / N_{0}$, where the rate is maximized over both the permissible $p_{0}$ and the permissible ring radius ratios.

Based on our search, we have found that shaping gains of up to $0.32 \mathrm{~dB}$ are achievable with the proposed shaping strategies. Fig. 4 shows the achievable information rates with shaping for 16-APSK and 32-APSK in AWGN, optimized over the shaping code and ring radius ratios. The results for 16-APSK show the benefit of using two shaping bits over just one shaping bit. From these curves, it is clear that if shaping is used at all, then it is advisable to use two shaping bits. The results for 32-APSK show that while 3 shaping bits is better than just 1 , using 2 shaping bits is actually worse than 1 . This is because using just 1 shaping bit segments the constellation into two natural subconstellations: One containing the two innermost rings and the other containing just the outer ring. On the other hand, 2 shaping bits creates an awkward partitioning that results in points in the middle ring being picked with different probabilities. For this reason, it is not advisable to use 2 shaping bits in the 32-APSK case. Furthermore, the incremental gain of using 3 shaping bits over 1 shaping bit is quite marginal, and it is recommended that 32-APSK systems use just 1 shaping bit. This result has an intuitive justification: With one shaping bit, the system will simply choose from the outer ring or the inner two rings. The results from Fig. 4 are tabulated for AWGN in Table I. For each value of $M$ and $g$, the tables show the rate $R$ for which shaping has the highest gain, along with the permissible values of $p_{0}$ and $\gamma$ that achieve the gain. The value of $\mathcal{E}_{b} / N_{0}$ for which the shaped constellation achieves rate $R$ is listed, where $\mathcal{E}_{b} / N_{0}=(1 / R)\left(\mathcal{E}_{s} / N_{0}\right)$ is the SNR per information bit. 
TABLE I

MINIMUM REQUIRED $\mathcal{E}_{b} / N_{0}$ IN AWGN FOR M-APSK WITH $g$ SHAPING BITS. THE OPTIMIZING $p_{0}$ AND $\gamma$ ARE SHOWN.

\begin{tabular}{|c|c||c|c|c|c|c|}
\hline $\mathrm{M}$ & $g$ & $R$ & $\mathcal{E}_{b} / N_{0}$ & gain & $p_{0}$ & $\gamma$ \\
\hline \multirow{2}{*}{16} & 1 & 3.09 & $4.714 \mathrm{~dB}$ & $0.091 \mathrm{~dB}$ & 0.623 & 2.70 \\
\cline { 2 - 7 } & 2 & 2.95 & $4.077 \mathrm{~dB}$ & $0.322 \mathrm{~dB}$ & 0.688 & 2.57 \\
\hline \multirow{2}{*}{32} & 1 & 3.88 & $5.915 \mathrm{~dB}$ & $0.265 \mathrm{~dB}$ & 0.716 & $\{2.64,4.64\}$ \\
\cline { 2 - 7 } & 2 & 4.06 & $6.517 \mathrm{~dB}$ & $0.175 \mathrm{~dB}$ & 0.623 & $\{2.53,4.30\}$ \\
\cline { 2 - 7 } & 3 & 3.89 & $5.898 \mathrm{~dB}$ & $0.310 \mathrm{~dB}$ & 0.656 & $\{2.53,4.30\}$ \\
\hline
\end{tabular}

TABLE II

PARAMETERS USED FOR THE 32-APSK SIMULATION.

\begin{tabular}{|c||c|c|c|c|}
\hline$\gamma$ & $R$ & $R_{c}$ & $R_{s}$ & $\mathcal{E}_{b} / N_{0}$ in $\mathrm{dB}$ \\
\hline \multirow{2}{*}{$2.64,4.64$} & 3.849 & $5000 / 6491$ & 1 & 6.092 \\
\cline { 2 - 5 } & 3.858 & $5000 / 6190$ & $7 / 9$ & 5.834 \\
\hline
\end{tabular}

\section{IMPLEMENTATION}

In this section, we compare the bit error performance of bit-interleaved coded APSK both with and without shaping. Because it is able to achieve high shaping gain with just one shaping bit, we focus on the case of 32-APSK with $g=1$. We let $R=3.85 \mathrm{bits} / \mathrm{symbol}$, which is close to the rate that achieves the highest shaping gain. At this $R$, we consider a system with a shaped constellation and a system with a uniform constellation. Table II shows the parameters that were used in the simulations. The table shows the ring radius ratios, overall code rate $R$, turbo code rate $R_{c}$, shaping code rate $R_{s}$, and value of $\mathcal{E}_{b} / N_{o}$ for which the constellation achieves information rate $R$. The row with $R_{s}=1$ corresponds to the uniform constellations. The turbo code is the one specified in the UMTS standard [11].

The receiver is based on [6], which describes two receiver implementations. In one implementation, extrinsic information is fed back from the decoder to the APSK dempper, while in another implementation, information is not fed back. In both implementations, information is exchanged between the turbo and shaping decoders. We refer to the first implementation as $B I C M-I D$, i.e. BICM with iterative demapping, while we refer to the second implementation as BICM. When a Gray mapping is employed, which is possible with PAM and QAM, there is little benefit to using BICM-ID [6]. However, with APSK, it is not possible to have a true Gray mapping, and as a consequence, there is some gain to using BICM-ID. In both cases, we consider 12 decoder iterations, where a full iteration includes an iteration of the shaping decoder, an iteration of the turbo decoder (one iteration for each of the constituent decoders), and, for the case of BICM-ID, one iteration of symbol demapping.

Bit error rate results for the AWGN channel are shown in Fig. 5. Both with and without shaping, BICM-ID provides a gain of about $0.7 \mathrm{~dB}$ over BICM. When using BICM, the shaping gain is about $0.6 \mathrm{~dB}$, and with BICM-ID, it is also about $0.6 \mathrm{~dB}$. This shaping gain is actually higher than the $0.265 \mathrm{~dB}$ gain predicted by the information-theoretical analysis (see Table I). This additional gain is most likely caused by the use of the shaping code and the inclusion of its decoder in the overall decoding loop. Essentially, the shaping code adds some additional coding gain over the gain of the turbo code.

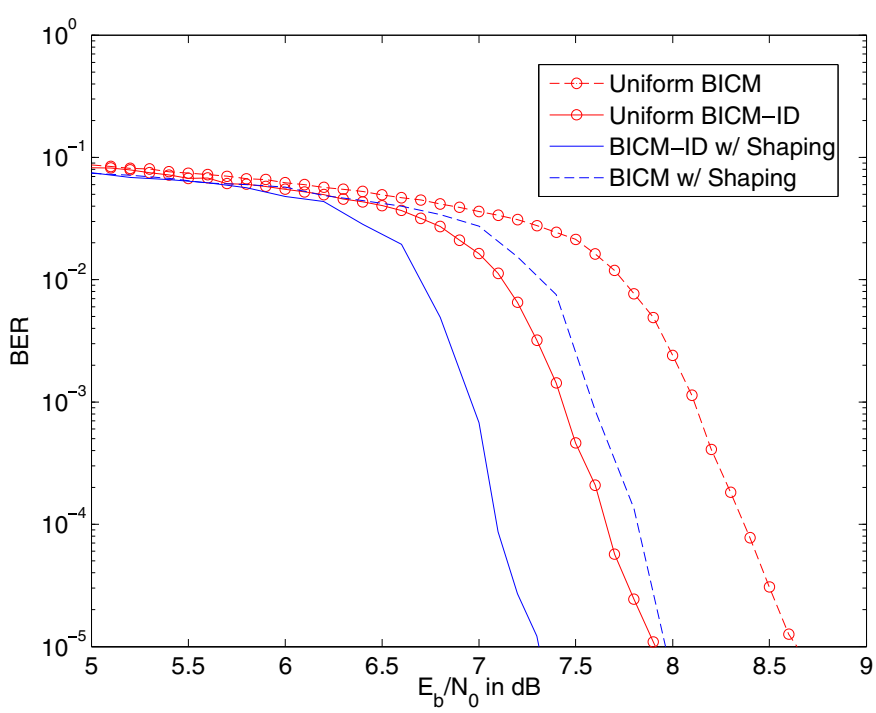

Fig. 5. Bit-error rate of 32-APSK in AWGN at rate $R=3.85$ bits/symbol.

\section{CONCLUSION}

APSK modulation is a good candidate for practical constellation shaping. The optimal constellation and shaping parameters may be determined by optimizing the information rate with respect to these parameters. Theoretical gains of up to $0.322 \mathrm{~dB}$ are achievable in the AWGN channel. In practice, a shaping gain of $0.6 \mathrm{~dB}$ is observed for 32-APSK from the AWGN bit error simulations. Future work should focus on the interaction with LDPC codes, since they are the codes used in the DVB-S2 standard.

\section{REFERENCES}

[1] European Telecommunications Standards Institute, "Digital video broadcasting (DVB) second generation: Framing structure, channel coding and modulation systems for broadcasting, interactive services, news gathering and other broad band satellite application," ETSI EN 302307 version 1.2.1, Aug. 2009.

[2] R. D. Gaudenzi, A. Guillén i Fàbregas, and A. Martinez, "Turbo-coded apsk modulations design for satellite broadband communications," Int. J. Satell. Commun. Network, vol. 24, pp. 261-281, May. 2006.

[3] K. Liolis and N. Alagha, "On 64-APSK constellation design optimization," in Proc. 10th Int. Workshop on Signal Processing for Space Communications (SPSC)., (Rhodes, Greece), Oct. 2008.

[4] A. R. Calderbank and L. H. Ozarow, "Nonequiprobable signaling on the gaussian channel," IEEE Trans. Inform. Theory, vol. 36, pp. 726-740, Jul. 1990.

[5] B. K. Khoo, Stéphane Y. Le Goff, and B. S. Sharif, "Bit-interleaved coded modulation with iterative decoding using constellation shaping," IEEE Trans. Commun., vol. 54, pp. 1517-1520, Sept. 2006.

[6] Stéphane Y. Le Goff, B. K. Khoo, and C. C. Tsimenidis, "Constellation shaping for bandwidth-efficient turbo-coded modulation with iterative receiver," IEEE Trans. Wireless Comm., vol. 6, pp. 2223-2233, Jun. 2007.

[7] R. Gallager, Information Theory and Reliable Communication. Wiley, 1968.

[8] R. E. Blahut, "Computation of channel capacity and rate distortion functions," IEEE Trans. Inform. Theory, vol. 18, pp. 460-473, Apr. 1972.

[9] G. Caire, G. Taricco, and E. Biglieri, "Bit-interleaved coded modulation," IEEE Trans. Inform. Theory, vol. 44, pp. 927-946, May 1998.

[10] P. E. McIllree, "Channel capacity calculations for m-ary n-dimensional signal sets," Master's thesis, The University of South Australia, Feb 1995.

[11] European Telecommunications Standards Institute, "Universal mobile telecommunications system (UMTS): Multiplexing and channel coding (FDD)," 3GPP TS 25.212 version 7.4.0, Jun. 2006. 